\title{
A Simple Insulated Monopole Sensor Technique for Determination of Moisture Content in Hevea Rubber Latex
}

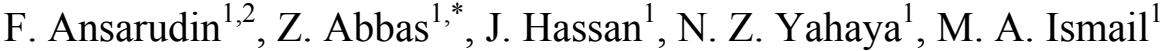 \\ ${ }^{1}$ Department of Physics, Faculty of Science, Universiti Putra Malaysia, 43400, Serdang, Selangor Malaysia. \\ *Corresponding author: za@science.upm.edu.my \\ ${ }^{2}$ Department of Electrical, Electronic and Systems Engineering, Faculty of Engineering and Built Environment, Universiti \\ Kebangsaan Malaysia, 43600 Bangi, Selangor, Malaysia, izah@vlsi.eng.ukm.my
}

\begin{abstract}
This paper describes the application of a SMA stub contact panel as an insulated monopole sensor for determination of moisture content in hevea rubber latex. Comparison results between measured and calculated reflection coefficient using King's model are presented. Calibration equations relating moisture content to phase shift have been established in the frequency range from 100 MHz to $5 \mathrm{GHz}$. The accuracy of the technique was $1.3 \%$ when compared to actual moisture content obtained using the gravimetric method.
\end{abstract}

Keywords: Reflection coefficient, phase shift, relative error

\section{INTRODUCTION}

$\mathrm{M}$ OISTURE CONTENT is one of the most important quantities for assessment of various food and agricultural products. Determination of moisture content (m.c.) is also necessary to calculate indirectly the solid content of the product that remains after total drying of the samples. Various techniques have been developed for the determination of moisture content in materials [1]. Moisture content can be determined by a variety of methods, but obtaining fast, accurate data is commonly a challenge especially if the required method is non-destructive.

In the rubber industry, the moisture content of freshly tapped natural rubber latex is usually between $40 \%$ and $65 \%$ [2-4]. Factors like the climatic conditions and tapping intensity will affect the concentration of Hevea brasiliensis latex. The lower the amount of water in fresh latex, the higher shall be the price. Bad practices such as addition of too much water for dilution could result in a dispute between the buyer and seller. Unfortunately, existing methods including the gravimetric method for determination of latex concentration are either laborious, inefficient, difficult, time consuming, or inaccurate.

In recent years, microwave measurement techniques have been proposed to determine the quality of fresh hevea latex. Material properties at microwave frequencies are usually described by the complex relative permittivity

$$
\varepsilon^{*}=\varepsilon^{\prime}-j \varepsilon^{\prime \prime}
$$

where the real part $\varepsilon^{\prime}$ or the relative dielectric constant is related to the ability of the material to store electrical energy, while the imaginary part $\varepsilon$ " or the loss factor represents an energy dissipating component. At microwave frequencies, the relative permittivity of water is higher than other materials. Hence, a small change in water content causes a relatively large change in the permittivity of the wet material. The advantage of the microwave method is in its independence from the level of salt content present in the material in contrary to low frequency techniques such as the capacitance method.

An experimental study was carried out on dielectric properties of the dry rubber content based on the microwave measurement technique [5]. The method was based on a short-circuited waveguide technique employing the two point method where the permittivity was calculated from measured guided wavelength determined from the distances between alternate minima. The method is laborious, time consuming, and the sample volume is small due to the restriction of the waveguide dimension. A dual frequency microstrip sensor [6] has been proposed to measure moisture content of hevea rubber latex at two frequencies in the Xband, $8.48 \mathrm{GHz}$ and $10.69 \mathrm{GHz}$. The technique is expensive, requiring two power diode detectors and a pair of dielectric resonators of different frequencies as signal sources. Later, an open ended coaxial sensor [7] operating at approximately $5 \mathrm{GHz}$ was used to determine moisture content in hevea latex. Except for the microcontroller, the measurement procedure and setup including the sensor and microwave circuitry was essentially based on a $500 \mathrm{MHz}$ reflectometer designed for moisture measurement of oil palm fruits [8] with extended applications in $[9,10]$. A calibration equation was used to determine the amount moisture content from the magnitude of the measured reflection coefficient of the open ended coaxial sensor, which in turn was used to predict the total solid content of hevea rubber latex. Unfortunately, the dynamic range of the magnitude of the reflection coefficient (0.75 to 0.95$)$ was too small to accurately predict the percentages of moisture content in the latex sample, especially in the presence of air bubbles. Thus, the technique is only suitable for subsurface measurement of moisture of a thin layer of latex due to its low electromagnetic field penetration depth. However, the penetration depth could be resolved probably by using a more dedicated instrument, such as TROTEC T600 Material Moisture Meter [11] enabling penetration to depths about $30 \mathrm{~cm}$. Nevertheless, it should also be noted that the maximum penetration depth is significantly reduced if the material that is to be measured is 
intensely saturated with moisture in the region near the surface. An alternative to the open ended coaxial sensor is the monopole antenna, which has been widely used in many applications such as in [12]. The monopole antenna sensor has also been used to determine moisture content in latex [13]. The monopole sensor is basically a blade monopole antenna designed for communication application at $2.4 \mathrm{GHz}$. A full immersion of the sensor in latex will allow higher penetration giving a high dynamic range of the reflection coefficient (almost from zero to near unity). The accuracy of the technique for determination of moisture content in latex was $3 \%$ when compared to the gravimetric method.

This paper presents the potential application of a commercially available stub contact panel as an insulated monopole sensor for the determination of moisture content in hevea rubber latex based on phase shift measurements.

\section{SuBJECT \& METHODS}

The pioneering work of King [14] on the analysis of insulated antenna was reported in 1974. Since then, several analytical and numerical models have been proposed but at the expense of high computational complexity. Also, the accuracy and applicability of the models have not been tested for semi-liquid materials like the hevea rubber latex samples. According to King's model, the total axial current in the insulated antenna shown in Fig. 1 can be expressed in the form

$$
I(z)=\frac{-i V_{o}}{Z_{c}}\left(\frac{\sin k_{a}\left(h_{a}-z\right)}{\cos k_{a} h_{a}}\right)
$$

where the characteristic impedance, $Z_{c}$ and the complex wave number, $\mathrm{k}_{\mathrm{a}}$ of antenna can be expressed specifically

$$
\begin{gathered}
Z_{c}=\frac{\zeta_{i} k_{a}}{2 \pi k_{i}}\left(\ln \frac{b}{a}\right) \\
k_{a}=k_{i}\left(1+\frac{H_{o}^{(1)}\left(k_{s} b\right)}{H_{1}^{(1)}\left(k_{s} b\right) \ln \left(\frac{b}{a}\right)}\right)
\end{gathered}
$$

The subscripts $s$ and $i$ represent the sample (hevea latex) and insulation medium, respectively. $\zeta_{i}$ is the wave impedance of insulating region

$$
\zeta_{i}=\frac{\omega \mu_{o}}{k_{i}}
$$

$H_{n}^{(1)}$ is a Hankel Function of order n, whilst the wave number, $\mathrm{k}$

$$
k=\left(\frac{2 \pi}{\lambda}\right) \sqrt{\varepsilon_{r}}
$$

where $\varepsilon_{\mathrm{r}}$ is the relative permittivity of material. The input admittance of the insulated monopole antenna (at $z=0$ ) is obtained from the ratio of the voltage, $V_{\mathrm{o}}$ to the current at the drive point (1), that is

$$
Y=\left(-\frac{i}{Z_{c}}\right) \tan \left(k_{a} h_{a}\right)
$$

The reflection coefficient of the material under test can be easily calculated as follows:

$$
\Gamma=\frac{1-Y}{1+Y}
$$

The insulated monopole sensor used in this work was a modification of a commercial standard SMA stub contact panel manufactured by Tyco Electronics. The $12.7 \mathrm{~mm}$ by $12.7 \mathrm{~mm}$ square flange served as the ground plane. The input port was a SMA female type with insulation material made of polytetrafluroethylene (PTFE). The protruding inner conductor of the stub was cut so that it had similar length $h_{a}$ with the insulated material, i.e., $15 \mathrm{~mm}$ as shown in Fig.1. The radius of the inner conductor $a$ and insulated material $b$ were $0.65 \mathrm{~mm}$ and $2.05 \mathrm{~mm}$, respectively. All the calibration and reflection coefficient measurements were carried out using an Agilent 5230A Performance Network Analyzer (PNA). The reflection coefficient values of the sensor at $\mathrm{z}=0$ were realized by applying the PNA's port extension technique.

\section{SAMPLE PREPARATION}

A series of solutions was prepared from the freshly tapped latex. These solutions were diluted with different volumes of deionized water to obtain different percentages of moisture content in latex. A total of about 50 samples of different percentages of m.c. were used in this study.

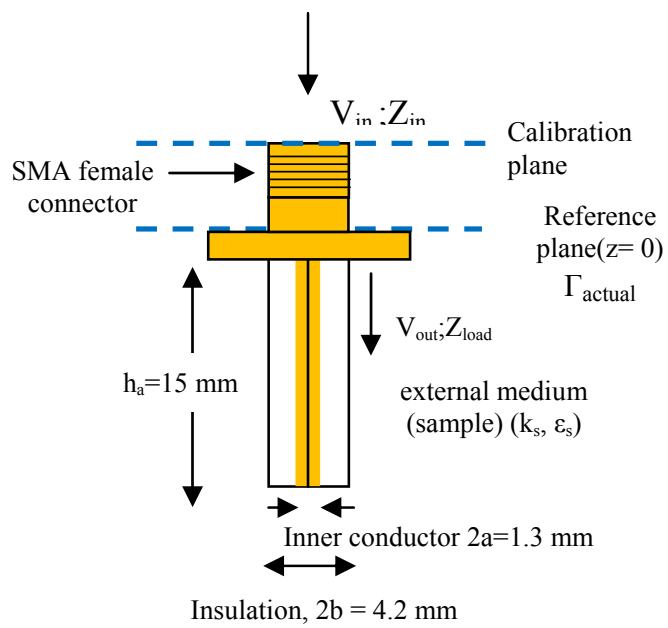

Fig.1. Dimensions of the insulated monopole sensor.

Ammonia was added to prevent the latex content from coagulation. The mass of ammonia is negligible, always less than $0.39 \%$ of the mass of the fresh latex [13-15]. The actual 
moisture content of rubber latex in percentage was obtained using the gravimetric method [16] and it was calculated specifically as:

$$
m . c(\%)=\frac{m_{w}-m_{d}}{m_{w}} \times 100
$$

where the $m_{w}$ and $m_{d}$ is the mass of wet and dry samples, respectively. The mass of fresh and diluted latex samples was initially recorded using electronic balance before being totally dried using a laboratory oven initially at $70{ }^{\circ} \mathrm{C}$ for 18 hours. Subsequently, the samples were dried at $105^{\circ} \mathrm{C}$ until a constant mass of difference of 0.0005 grams between the results of duplicates was obtained, which could take an extra 6 hours to complete the drying operation. The petri dishes were weighed and left in the desiccator at $26{ }^{\circ} \mathrm{C}$ as soon as it has cooled to laboratory temperature. These operations must be done as quickly as possible to avoid any appreciable change in moisture content.

\section{RESULTS AND DISCUSSION}

Comparison between measured and calculated magnitude and phase of the reflection coefficient of the sensor for typical fresh latex with moisture content $42.47 \%$ is shown in Fig.2a and $2 \mathrm{~b}$, respectively. It can be clearly seen from the graphs that the calculated magnitudes of the reflection coefficient using King's model were in good agreement with the measured data only below $3 \mathrm{GHz}$. This was probably due to the use of approximate numerical calculation of electric field in the model that limits the accuracy [14] of the fields near the surface of the insulation, especially at very high frequencies.

It should be noted that the results are based on the geometrical dimensions of the sensor fabricated from a commercial stub contact panel shown in Fig.1. Higher agreement can be expected by fabricating a sensor of optimum dimensions for latex measurement. Due to the geometrical dimensions of the sensor, especially finite ground plane and thick insulated medium, the variations in both the magnitude and phase of reflection coefficient were almost overlapping for all latex samples ranging from $36.40 \%$ to $82.45 \%$ as shown in Fig.3. Also shown in the graphs are the results for water and the unloaded sensor (air).

This difficulty can be resolved by plotting the phase shift, i.e., the difference in phase between the unloaded and loaded sensor at different percentages of m.c. Theoretically, the change in m.c. will result in change in permittivity of the samples which in turn will change the phase shift of the probing electromagnetic waves [17]. The phase shift is a function of the permittivity, density, and thickness of the sample. In this work, the samples were infinitely thick. Thus, the values of permittivity and density were dictated by the percentage of m.c. In contrast, the magnitude of reflection coefficient of the sensor is a combination of reflection of various sources such as permittivity of the sample, multiple reflection at the input impedance at $\mathrm{z}=0$ as well as along the cylindrical rod and effect of finite ground plane.

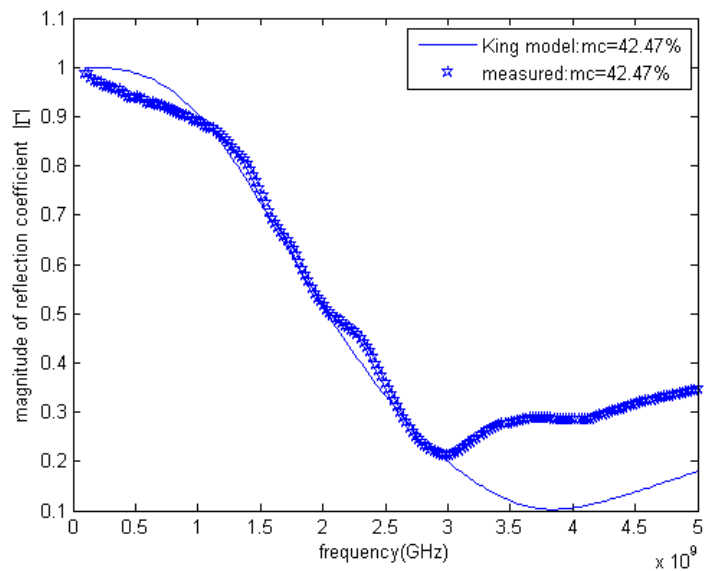

(a)

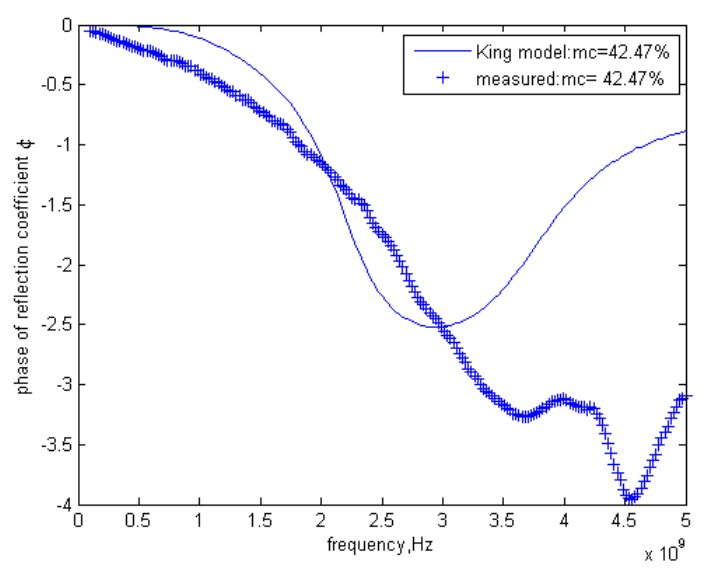

(b)

Fig.2. Variation in (a) magnitude, (b) phase of reflection coefficient for latex with $42.47 \%$ m.c.

The calibration equations relating moisture content to phase shift at various frequencies listed in Table 1 were found by means of regression analysis. The highest correlation (0.8955) and highest sensitivity (-779.94) were obtained at $0.52 \mathrm{GHz}$. The effect of m.c. on the phase shift at $0.52 \mathrm{GHz}$ is illustrated in Fig.4.

Table 1. Calibration equation relating moisture content to $\Delta \phi$

\begin{tabular}{cclc}
$\begin{array}{c}\text { Frequency } \\
(\mathrm{GHz})\end{array}$ & $\begin{array}{c}\text { Regression } \\
\text { Coefficient } \\
\left(\mathrm{R}^{2}\right)\end{array}$ & \multicolumn{1}{c}{ Equation } & $\begin{array}{c}\text { Mean } \\
\text { Relative } \\
\text { Error }\end{array}$ \\
\hline 0.52 & 0.8955 & $\mathrm{MC}=-779.94 \Delta \phi-231.51$ & 0.013 \\
1.0 & 0.8804 & $\mathrm{MC}=-192.97 \Delta \phi-99.047$ & 0.714 \\
1.5 & 0.5658 & $\mathrm{MC}=-153.19 \Delta \phi-150.29$ & 0.182 \\
1.8 & 0.8204 & $\mathrm{MC}=-44.741 \Delta \phi-19.876$ & 0.237 \\
2.0 & 0.0003 & $\mathrm{MC}=-1.59 \Delta \phi+60.451$ & 0.261 \\
2.4 & 0.3057 & $\mathrm{MC}=46.97 \Delta \phi+188.8$ & 0.193 \\
3.0 & 0.6168 & $\mathrm{MC}=37.18 \Delta \phi+186.38$ & 0.113 \\
3.5 & 0.8616 & $\mathrm{MC}=64.32 \Delta \phi+283.47$ & 0.158 \\
4.0 & 0.8657 & $\mathrm{MC}=70.18 \Delta \phi+297.73$ & 0.167 \\
4.5 & 0.8660 & $\mathrm{MC}=80.89 \Delta \phi+411.26$ & 0.147 \\
5.0 & 0.7662 & $\mathrm{MC}=95.97 \Delta \phi+434.96$ & 0.624 \\
\hline
\end{tabular}




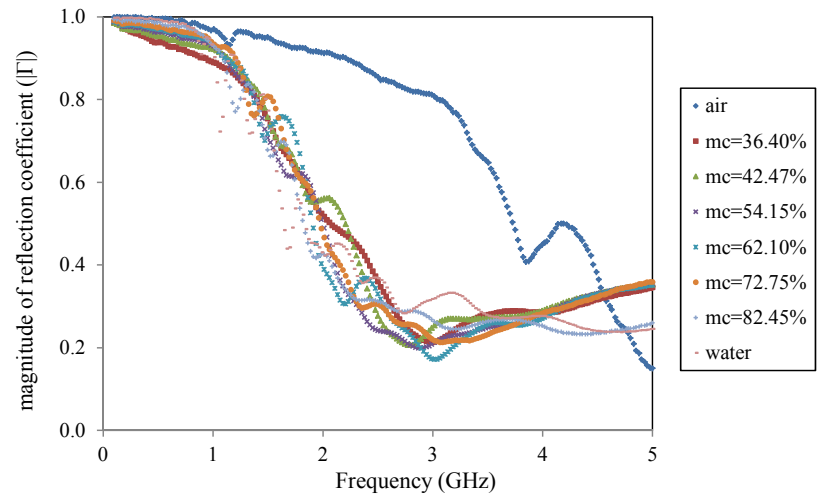

(a)

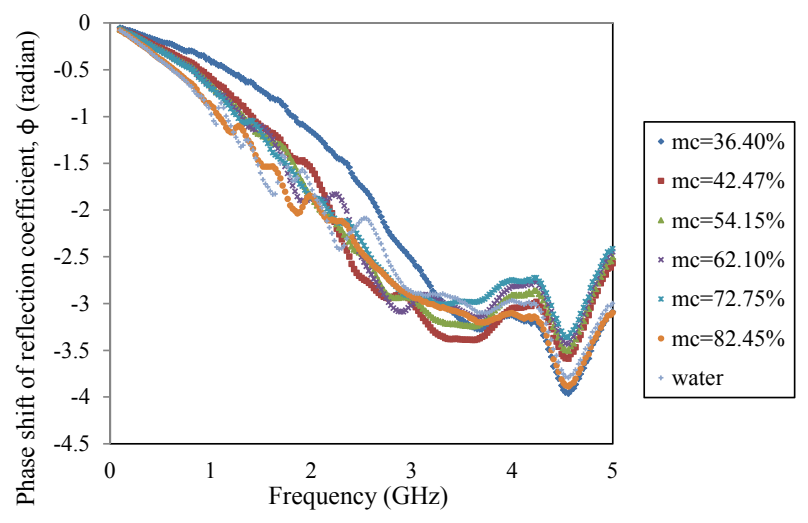

(b)

Fig.3. Variation in (a) magnitude (b) phase of reflection coefficient for unloaded sensor(air), water and samples with different percentages m.c. with frequency.

Table 1 also suggests that the regression coefficient values were initially high at low frequencies before slowly reducing to a minimum value about $2.0 \mathrm{GHz}$ before rising again at higher frequencies. This phenomenon is almost similar to the loss factor of hevea rubber latex measured using the Agilent 85070B Dielectric Probe Kit shown in

Fig.5, while the relative dielectric constant $\varepsilon^{\prime}$ decreases slowly with increasing frequency for all samples of different percentages m.c., however, the loss factor $\varepsilon$ " initially decreases with frequency until $1.8 \mathrm{GHz}$ before increasing almost proportionally with increasing frequencies. At frequencies below $1.8 \mathrm{GHz}$, the higher the amount of moisture content the lower the values of $\varepsilon^{\prime \prime}$. In contrast, the higher the amount of moisture content, the higher shall be the values of $\varepsilon$ "above $1.8 \mathrm{GHz}$. This critical frequency is typical for semi-liquid materials like hevea rubber latex which exhibit more conductive behavior for frequencies lower than $2.0 \mathrm{GHz}$ [15]. At this critical frequency, the electrical properties of latex are predominantly influenced by the conductive losses and dipole orientation in water molecules below and above $1.8 \mathrm{GHz}$, respectively. It should be noted that the critical frequency was not observed for totally dry samples (not shown in the figure) having almost constant $\varepsilon^{\prime}(2.7$ to 2.7$)$ and $\varepsilon^{\prime \prime}(0.16$ to 0.17$)$ for the whole frequency range from $100 \mathrm{MHz}$ to $5 \mathrm{GHz}$. Since the phase shift is also affected by the sensor' geometry, especially the ground plane, it is assumed that the low regression coefficient at $2.0 \mathrm{GHz}$ was due to the uncertainties attributed to the combined effects of both the sensor's geometry and sample properties.

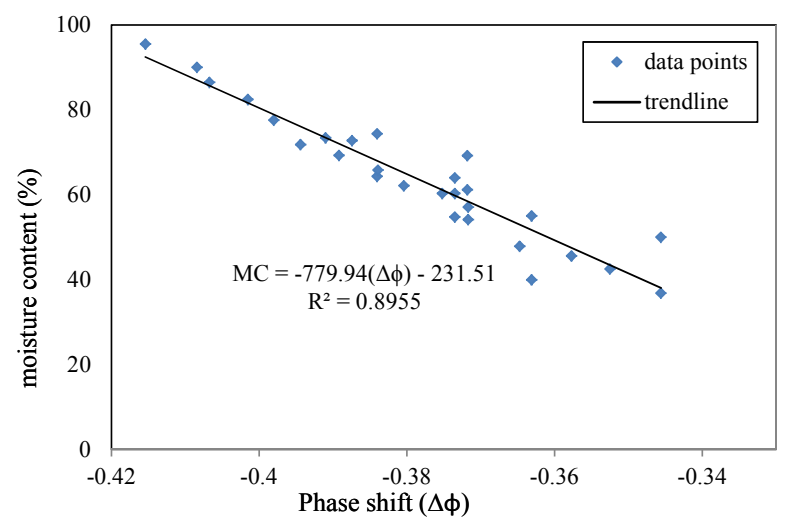

Fig.4. Relationship between phase shifts $(\Delta \phi)$ and Moisture content at $0.52 \mathrm{GHz}$

An equation with high regression coefficient does not necessarily produce high accuracy prediction results. Accurate results can only be validated by comparing the predicted m.c. results obtained indirectly from the reflection measurements with the actual m.c. found using the gravimetric method. The relative error in the predicted moisture content with respect to the actual moisture content obtained using the gravimetric method was based on the following equation:

$$
\text { Relative Error }=\left|\frac{\mathrm{mc}_{\text {actual }}-\mathrm{mc}_{\text {predicted }}}{\mathrm{mc}_{\text {actual }}}\right|
$$

The calibration equations in Table1 were used to predict the amount of m.c. based on the measured phase shift on a new set of 37 latex samples of various percentages of m.c. The mean relative error results are also shown in Table1. The most accurate equation to predict moisture content in hevea latex based on the measurement of the phase shift of the reflection coefficient with the geometrical configuration illustrated in Fig. 1 was at $0.52 \mathrm{GHz}$, i.e.,

$$
M C=-779.94 \Delta \phi-231.51
$$

where $\mathrm{MC}$ is the predicted moisture content based on the measured phase shift $\Delta \phi$. The sensitivity of the sensor was $-779.94 \% \mathrm{mc} / \mathrm{rad}$, i.e., every phase shift measurement change of $0.001 \mathrm{rad}$ represents a change of $0.779 \%$ m.c. The mean relative error percentage when using equation (10) was approximately $\pm 1.3 \%$. Fig. 6 directly compares the predicted and actual m.c. The very high sensitivity value 0.9933 suggests almost perfect one-to-one correspondence between the predicted and actual m.c. 


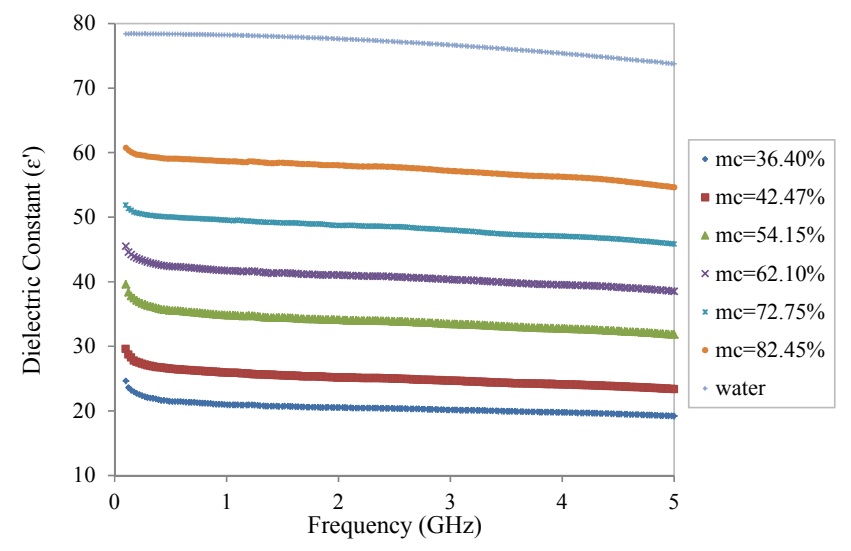

(a)

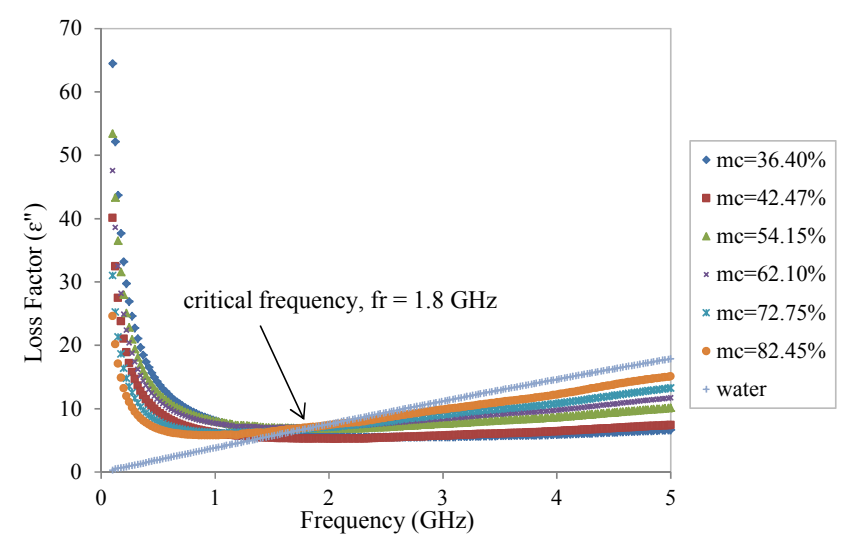

(b)

Fig.5. (a) Relative Dielectric Constant ( $\left.\varepsilon^{\prime}\right)$ (b) Loss Factor $\left(\varepsilon^{\prime \prime}\right)$ for various percentages of moisture content.

Similar accurate measurement results at the vicinity of $0.52 \mathrm{GHz}$ have been reported in [15] and other types of moist materials [1,17]. However, the exact causes are currently unknown, though several theories have been postulated such as the relaxation frequency of the second bound water layer at $0.5 \mathrm{GHz},[18]$. Another possible reason could be due to the sample unique properties where the most pronounced difference between free and bound water is located in the frequency domain from $100 \mathrm{MHz}$ to $1 \mathrm{GHz}$ with a maximum at about $500 \mathrm{MHz},[19]$.

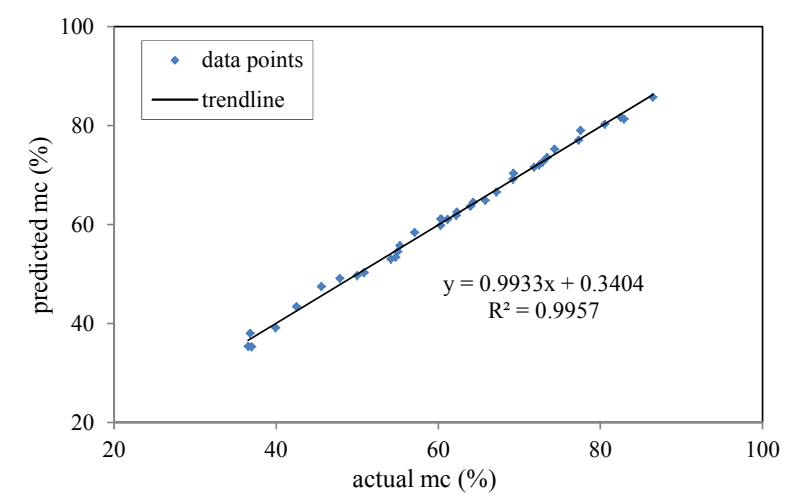

Fig.6. Direct comparison between predicted m.c. and actual m.c. at $0.52 \mathrm{GHz}$ using equation (9) and gravimetric method, respectively.

\section{CONCLUSION}

The results indicate that the amount of moisture content in hevea latex can be determined with excellent accuracy using a SMA stub contact panel as an insulated monopole sensor. The major benefit is that, unlike alternative techniques, it provides a measurement method that is simultaneously very quick, simple, cheap, and nondestructive.

\section{ACKNOWLEDGEMENT}

The authors gratefully acknowledge the Scholarship Division of Ministry of Higher Education, Malaysia for supporting this work.

\section{REFERENCES}

[1] Kupfer, K. (ed.) (2005). Electromagnetic Aquametry: Electromagnetic Wave Interaction with Water and Moist Substances. Springer.

[2] Poh, K.W. (1989). Developments of natural rubber latex. Production, properties, stability. Elastomerics, 12 (12), 12-15.

[3] Tomazic-Jezic, V.J., Beezhold, D.H., Hashim, H., Palosuo, T., Raulf-Heimsoth, M., Swanson, M., Hamilton, R.G. (2004). Performance of methods for the measurement of natural rubber latex (NRL) proteins, antigens andallergens. Journal of Allergy and Clinical Immunology, 113 (78).

[4] Chin, H.C. (1979). RRIM Training Manual on Analytical Chemistry. Research Institute Malaysia, Kuala Lumpur, 67.

[5] Jayanthy, T., Sankaranarayanan, P.E. (2005). Measurement of dry rubber content in latex using microwave technique. Measurement Science Review, 5 (3), 50-54.

[6] Ghretli, M., Khalid, K., Grozescu, I.V., Sahri, H., Abbas, Z. (2007). Dual frequency microstrip antenna sensor for water content measurements independent of temperature variation. Measurement Science Technology, 18, 1054-1060.

[7] Mohammadi, A., Khalid, K., Bafti, P.S., Homaiee, M. (2012). Development of microcontroller-based microwave system to measure solid content of hevea rubber latex. Measurement, 45 (3), 571-579.

[8] Kim, L.K (2004). Development of a $500 \mathrm{MHz}$ Reflectometer Using Stripline Coupler for Moisture Measurement of Oil Palm Fruits. Masters thesis, Universiti Putra Malaysia, 61-87.

[9] Yeow, Y.K., Abbas, Z., Khalid, K. (2010). Application of microwave moisture sensor for determination of oil palm fruits ripenes. Measurement Science Review, 10 (1), 7-14.

[10] Jusoh, A., Abbas, Z., Azmi, B.Z., Hassan, J., Meng, C.E., Ahmad, A.F. (2011). A simple procedure to determine complex permittivity of moist materials using standard commercial sensor. Measurement Science Review, 11 (1), 19-22.

[11] Rouane, A., Koutiche, D. (2012). Characterization of microwave antennas for intracardiac ablation frequencies 915 and $2450 \mathrm{MHz}$. Measurement Science Review, 12 (1), 34-39. 
[12] Trotec. (2009). T600 operating manual version 1. Trotec GmbH \&Co., Germany.

[13] Ali, A.H., Abbas, Z., Hassan, J., Jusoh, A., Zahari, R.M. (2011). Microwave antenna sensing technique for determination of moisture content in hevea latex. Australian Journal of Crop Science, 5 (11), 13261333.

[14] King, R.W.P., Lee, K.M., Glenn, S.S. (1974). Insulated linear antenna: Theory and experiment. Journal of Applied Physics, 45 (4), 1688-1697.

[15] Jumiah, H., Khalid, K., Daud, W.M. (1997). Microwave dielectric properties of hevea rubber latex in the temperature range of $-30^{\circ} \mathrm{C}$ to $50^{\circ} \mathrm{C}$. Pertanika Journal Science \& Technology, 5 (2), 179-190.
[16] MS 466: Methods of Sampling and Testing Field Latex. First Revision. (1987). Standard \& Research Institute of Malaysia.

[17] Nyfors, E., Vainikainen, P. (1989). Industrial Microwave Sensors. Norwood, MA: Artech House.

[18] Or, D., Wraith, J.M. (1999). Temperature effects on bulk dielectric permittivity measured by time domain reflectometry: A physical model. Water Resources Research, 35, 371-383.

[19] Pyper, J.W., Buettner, H.M., Cerjan, C.J., Hallam, J.S., King, R.J. (1985). The measurement of bound and free moisture in organic materials by microwave methods. In Proceedings of the International Symposium on Moisture \& Humidity, 15-18 April, 1985.

Received July 05, 2012. Accepted November 26, 2012. 